\title{
Transport coefficients from the boson Uehling-Uhlenbeck equation
}

\author{
Erich D. Gust* and L. E. Reichl ${ }^{\dagger}$ \\ The Center for Complex Quantum Systems, The University of Texas at Austin, Austin, Texas 78712 \\ (Received 6 August 2012; published 15 April 2013)
}

\begin{abstract}
Expressions for the bulk viscosity, shear viscosity, and thermal conductivity of a quantum degenerate Bose gas above the critical temperature for Bose-Einstein condensation are derived using the Uehling-Uhlenbeck kinetic equation. For contact potentials and hard sphere interactions, the eigenvalues (relaxation rates) of the Uehling-Uhlenbeck collision operator have an upper cutoff. This cutoff requires summation over all discrete eigenvalues and eigenvectors of the collision operator when computing transport coefficients. We numerically compute the shear viscosity and thermal conductivity for any boson gas that interacts via a contact potential. We find that the bulk viscosity of the degenerate boson gas remains identically zero, as it is for the classical gas.
\end{abstract}

DOI: 10.1103/PhysRevE.87.042109

PACS number(s): 51.10.+y, 67.10.Jn, 51.20.+d

\section{INTRODUCTION}

Kinetic equations provide a means to derive microscopic expressions for the transport coefficients appearing in the equations of fluid hydrodynamics. The transport coefficients for dilute gases at high temperature can be computed using the Boltzmann equation. However, when the temperature is lowered enough that quantum degeneracy begins to affect the behavior of the gas, one must use the Uehling-Uhlenbeck (U-U) equation [1,2], which is a semiclassical extension of the Boltzmann equation that accounts for the quantum statistics of the particles.

There are two traditional approaches to computing transport coefficients; the method outlined by Chapman and Enskog [3-6], and a method due to Resibois [7], which directly uses the microscopic hydrodynamic modes of the system. In subsequent sections, we use the Resibois method to compute the transport coefficients of dilute degenerate boson gases, and we give explicit numerical values for their transport coefficients just above the Bose-Einstein condensation temperature. Although we focus on boson gases, it is worth noting that there has been interest recently in the viscosity of some exotic systems whose relaxation is also governed by the U-U equation. These include the aftermath of high-energy collisions, and recent predictions of a universal limit on the ratio of viscosity and entropy density [3,8-11].

The Resibois method relates the transport coefficients to the spectral decomposition of the collision operator, and the discrete eigenvalues (relaxation rates) of the collision operator are central. For gases that interact via hard-core potentials or contact potentials, the eigenvalues of the Boltzmann and the Uehling-Uhlenbeck collision operators have a known upper cutoff [12] and the eigenvalues rapidly converge towards this value. When an upper cutoff exists, the contribution to the transport coefficients from higher eigenmodes can not be neglected. For gases that interact via a softer potential, such as $V(r) \sim \frac{1}{r^{n}}$ for $n>4$, no cutoff exists in the discrete spectrum of the collision operator and simple approximations can be used in obtaining transport coefficients. In subsequent sections, we obtain exact values for the transport coefficients of classical

\footnotetext{
*egust@physics.utexas.edu

${ }^{\dagger}$ reichl@austin.utexas.edu
}

hard sphere gases and ultracold boson gases, and we show how the Eucken number $[6,13]$ of classical gases changes as the interaction varies from that of a Maxwell gas $(n=4)$ to a hard sphere interaction $(n \rightarrow \infty)$, which is equivalent to a contact interaction at low temperature.

Typically, in the past, the transport coefficients of dilute classical hard sphere gases have been obtained by truncating a Sonine polynomial expansion at low order $[4,6,14]$. As we will show, this truncation neglects the effect of the upper cutoff on collision operator eigenvalues and gives an Eucken number that differs little from that of a classical Maxwell gas.

In Sec. II we introduce the U-U equation and in Sec. III, we linearize it and introduce an abstract velocity-space basis that will be used for calculation. In Sec. IV, we derive microscopic expressions for the hydrodynamic frequencies by relating them to the collision operator, and use them to obtain explicit expressions for the bulk viscosity, thermal conductivity, and shear viscosity of the gas. We also discuss the proper treatment of the eigenvalue "cutoff" that is present for hard sphere and contact interactions. In Sec. V, we present the results of our numerical calculation of the transport coefficients and justify our treatment of the eigenvalue cutoff. We conclude by summarizing our results in Sec. VI.

\section{UEHLING-UHLENBECK EQUATION}

We consider a dilute gas of noncondensed bosons that interact via a contact potential $U(\mathbf{r})=U_{0} \delta^{3}(\mathbf{r})$, where $U_{0}=$ $4 \pi \hbar^{2} a / m, \hbar$ is Planck's constant, $a$ is the $s$-wave scattering length, and $m$ is the mass of a particle. The derivation of a kinetic equation for this system is discussed in Ref. [15]. Above the critical temperature for Bose-Einstein condensation, the dynamics of the gas is governed by the Uehling-Uhlenbeck (U-U) kinetic equation, which can be written

$$
\frac{\partial f_{1}}{\partial t}+\frac{\mathbf{p}_{1}}{m} \cdot \nabla_{\mathbf{r}} f_{1}=-\mathcal{C}\left[f_{1}\right],
$$

where $\mathbf{p}_{1}$ and $\mathbf{r}$ are momentum and position of the bosons, respectively, and $f_{1}=f\left(\mathbf{r}, \mathbf{p}_{1}, t\right)$ is the phase space number density of bosons in the phase space volume $\mathbf{p}_{1} \rightarrow \mathbf{p}_{1}+d \mathbf{p}_{1}$ and $\mathbf{r} \rightarrow \mathbf{r}+d \mathbf{r}$ at time $t$. It is normalized so that $N=$ $\int d \mathbf{r} \int \frac{d \mathbf{p}_{1}}{(2 \pi \hbar)^{3}} f\left(\mathbf{r}, \mathbf{p}_{1}, t\right)$, where $N$ is the average number of particles in the gas. 
The quantity $\mathcal{C}\left[f_{1}\right]$ is the collision integral and is defined

$$
\begin{aligned}
\mathcal{C}\left[f_{1}\right]= & \frac{a^{2}}{m^{2} \pi^{3} \hbar^{3}} \int d \mathbf{p}_{2} d \mathbf{p}_{3} d \mathbf{p}_{4} \delta^{3}\left(\mathbf{p}_{1}+\mathbf{p}_{2}-\mathbf{p}_{3}-\mathbf{p}_{4}\right) \\
& \times \delta\left(\epsilon_{1}+\epsilon_{2}-\epsilon_{3}-\epsilon_{4}\right)\left[f_{1} f_{2}\left(1+f_{3}\right)\left(1+f_{4}\right)\right. \\
& \left.-\left(1+f_{1}\right)\left(1+f_{2}\right) f_{3} f_{4}\right]
\end{aligned}
$$

where $\epsilon_{1}=p_{1}^{2} /(2 m)$.

The collision integral explicitly conserves particle number, momentum, and energy. This can be seen from the five integrals $\int d \mathbf{p} \mathcal{C}[f(\mathbf{r}, \mathbf{p}, t)]=0, \int d \mathbf{p p} \mathcal{C}[f(\mathbf{r}, \mathbf{p}, t)]=0$, and $\int d \mathbf{p} p^{2} \mathcal{C}[f(\mathbf{r}, \mathbf{p}, t)]=0$. The stationary state (long-time global equilibrium) solution of the U-U equation is the Bose-Einstein distribution

$$
f^{0}\left(\mathbf{p}_{1}\right)=\left(\exp \left[\frac{\epsilon_{1}-\mu}{k_{B} T}\right]-1\right)^{-1},
$$

where $T$ is the equilibrium temperature in Kelvin, $\mu$ is the equilibrium chemical potential, and $k_{B}$ is Boltzmann's constant. Integrating $f^{0}\left(\mathbf{p}_{1}\right)$ over momentum gives the equilibrium particle density $n_{0}$ of the gas

$$
n_{0}=\int \frac{d \mathbf{p}_{1}}{(2 \pi \hbar)^{3}} f^{0}\left(\mathbf{p}_{1}\right)=\frac{1}{\lambda_{T}^{3}} \operatorname{Li}_{3 / 2}(z),
$$

where $\lambda_{T}=\sqrt{\frac{2 \pi \hbar^{2}}{m k_{B} T}}$ is the thermal wavelength, $z=e^{\mu /\left(k_{B} T\right)}$ is the fugacity and $\mathrm{Li}_{3 / 2}(z)$ is a polylogarithm. Polylogarithms appear repeatedly for degenerate gases and are defined by

$$
\mathrm{Li}_{s}(z)=\frac{1}{\Gamma(s)} \int_{0}^{\infty} d t \frac{z t^{s-1}}{\mathrm{e}^{t}-z} .
$$

For simplicity we will use the notation $\sigma_{n}=\operatorname{Li}_{\frac{n+1}{2}}(z)$.

It is useful to introduce a dimensionless momentum $\mathbf{c}_{1}=\mathbf{p}_{1} /\left(m v_{T}\right)$ where $v_{T}=\sqrt{2 k_{B} T / m}$. Then the UehlingUhlenbeck equation can be written

$$
\frac{\partial f_{1}}{\partial t}+v_{T} \mathbf{c}_{1} \cdot \nabla_{\mathbf{r}} f_{1}=-\gamma \mathcal{C}^{\prime}\left[f_{1}\right]
$$

where the dimensionless collision integral $\mathcal{C}^{\prime}\left[f_{1}\right]$ is given by

$$
\begin{aligned}
\mathcal{C}^{\prime}\left[f_{1}\right]= & \frac{1}{z \pi^{2}} \int d \mathbf{c}_{2} d \mathbf{c}_{3} d \mathbf{c}_{4} \delta^{3}\left(\mathbf{c}_{1}+\mathbf{c}_{2}-\mathbf{c}_{3}-\mathbf{c}_{4}\right) \\
& \times \delta\left(c_{1}^{2}+c_{2}^{2}-c_{3}^{2}-c_{4}^{2}\right)\left[f_{1} f_{2}\left(1+f_{3}\right)\left(1+f_{4}\right)\right. \\
& \left.-\left(1+f_{1}\right)\left(1+f_{2}\right) f_{3} f_{4}\right],
\end{aligned}
$$

and $f_{1}=f\left(\mathbf{r}, \mathbf{c}_{1}, t\right)$. The overall rate constant is

$$
\gamma=\frac{8 m a^{2} z\left(k_{B} T\right)^{2}}{\pi \hbar^{3}}
$$

which now depends on the fugacity and equilibrium temperature.

\section{LINEARIZED U-U EQUATION}

In computing transport coefficients, it is sufficient to consider the relaxation of the gas when it is close to equilibrium. In that case, the distribution function $f\left(\mathbf{r}, \mathbf{c}_{1}, t\right)$ will be a slowly varying function of $\mathbf{r}$, and will be close to its equilibrium value. We can then linearize the U-U equation by writing $f\left(\mathbf{r}, \mathbf{c}_{1}, t\right)=f_{1}^{0}+f_{1}^{0}\left(1+f_{1}^{0}\right) \Phi\left(\mathbf{r}, \mathbf{c}_{1}, t\right)$, where $f_{1}^{0}=z /\left(e^{c_{1}^{2}}-z\right)$ and $\Phi\left(\mathbf{r}, \mathbf{c}_{1}, t\right)$ contains information about the small deviations from equilibrium and satisfies $|\Phi| \ll 1$. We substitute this expression for $f\left(\mathbf{r}, \mathbf{c}_{1}, t\right)$ into (7) and neglect terms of quadratic and higher order in $\Phi$ and obtain

$$
\frac{\partial \Phi_{1}}{\partial t}+v_{T} \mathbf{c}_{1} \cdot \nabla_{\mathbf{r}} \Phi_{1}=-\gamma C\left[\Phi_{1}\right]
$$

where $\Phi_{1}=\Phi\left(\mathbf{r}, \mathbf{c}_{1}, t\right)$ and

$$
\begin{aligned}
C\left[\Phi_{1}\right]= & \frac{1}{z \pi^{2}\left(1+f_{1}^{0}\right)} \int d \mathbf{c}_{2} d \mathbf{c}_{3} d \mathbf{c}_{4} \delta^{3}\left(\mathbf{c}_{1}+\mathbf{c}_{2}-\mathbf{c}_{3}-\mathbf{c}_{4}\right) \\
& \times \delta\left(c_{1}^{2}+c_{2}^{2}-c_{3}^{2}-c_{4}^{2}\right) f_{2}^{0}\left(1+f_{3}^{0}\right)\left(1+f_{4}^{0}\right) \\
& \times\left[\Phi_{1}+\Phi_{2}-\Phi_{3}-\Phi_{4}\right]
\end{aligned}
$$

is the linearized collision operator.

Since Eq. (9) is linear, we may take the Fourier transform of both the space and time dependence of (9) to obtain

$$
-i \omega \phi_{1}+i v_{T}\left(\mathbf{k} \cdot \mathbf{c}_{1}\right) \phi_{1}=-\gamma C\left[\phi_{1}\right]
$$

where $\phi_{1}=\phi\left(\mathbf{k}, \mathbf{c}_{1}, \omega\right)=\int d \mathbf{r} \int d t \Phi\left(\mathbf{r}, \mathbf{c}_{1}, t\right) e^{-i \mathbf{k} \cdot \mathbf{r}+i \omega t}$. Equation (11) governs the relaxation of deviations from equilibrium with wave vector $\mathbf{k}$ and frequency $\omega$.

\section{A. Momentum basis}

Since (11) is linear, deviations $\phi\left(\mathbf{k}, \mathbf{c}_{1}, \omega\right)$ will not be coupled to those of different wave vectors or frequencies and we may suppress the dependence of $\phi\left(\mathbf{k}, \mathbf{c}_{1}, \omega\right)$ on $\mathbf{k}$ and $\omega$. We can greatly simplify the notation in the following calculations by introducing an abstract vector $|\phi\rangle$ and making the interpretation $\phi\left(\mathbf{c}_{1}\right)=\left\langle\mathbf{c}_{1} \mid \phi\right\rangle$, where $\left|\mathbf{c}_{1}\right\rangle$ represents an abstract momentum basis. We define the inner product between two abstract vectors as

$$
\langle\chi \mid \phi\rangle \equiv \int d \mathbf{c}_{1} w\left(c_{1}\right) \chi^{*}\left(\mathbf{c}_{1}\right) \phi\left(\mathbf{c}_{1}\right),
$$

where the weighting factor $w\left(c_{1}\right)$ is defined

$$
w\left(c_{1}\right)=\frac{1}{\pi^{3 / 2} \sigma_{0}} f^{0}\left(c_{1}\right)\left[1+f^{0}\left(c_{1}\right)\right] .
$$

If $C[\phi]$ is interpreted as $\left\langle\mathbf{c}_{1}|\hat{C}| \phi\right\rangle$, this definition of the weighting function makes the collision operator symmetric in the sense that $\left\langle\mathbf{c}_{1}|\hat{C}| \mathbf{c}_{2}\right\rangle=\left\langle\mathbf{c}_{2}|\hat{C}| \mathbf{c}_{1}\right\rangle$. In the momentum basis, the collision operator $\hat{C}$ can then be expressed as

$$
\hat{C}=\int d \mathbf{c}_{1} \int d \mathbf{c}_{5} w\left(c_{1}\right) w\left(c_{5}\right)\left|\mathbf{c}_{1}\right\rangle C\left(\mathbf{c}_{1}, \mathbf{c}_{5}\right)\left\langle\mathbf{c}_{5}\right|,
$$

where $C\left(\mathbf{c}_{1}, \mathbf{c}_{5}\right)=\left\langle\mathbf{c}_{1}|\hat{C}| \mathbf{c}_{5}\right\rangle$ is the "kernel function" of $\hat{C}$.

It will be convenient to introduce an orthonormal "angle" basis, $|c, l, m\rangle$, which is defined by

$$
\left\langle\mathbf{c}_{1} \mid c, l, m\right\rangle=\frac{1}{c_{1} \sqrt{w\left(c_{1}\right)}} \delta\left(c_{1}-c\right) Y_{l}^{m}\left(\theta_{1}, \phi_{1}\right) .
$$

The collision operator can be expressed in the angle basis as

$$
\begin{aligned}
\hat{C}= & 2 \pi \int_{0}^{\infty} d c_{1} \int_{0}^{\infty} d c_{2} \sum_{l, m}\left|c_{1}, l, m\right\rangle \\
& \times c_{1} c_{2} \sqrt{w\left(c_{1}\right) w\left(c_{2}\right)} C^{l}\left(c_{1}, c_{2}\right)\left\langle c_{2}, l, m\right|,
\end{aligned}
$$


where

$$
C^{l}\left(c_{1}, c_{2}\right)=\int_{-1}^{1} d\left(\hat{\mathbf{c}}_{1} \cdot \hat{\mathbf{c}}_{2}\right) C\left(\mathbf{c}_{1}, \mathbf{c}_{2}\right) P_{l}\left(\hat{\mathbf{c}}_{1} \cdot \hat{\mathbf{c}}_{2}\right)
$$

and $P_{l}$ is a Legendre polynomial. The function $C^{l}\left(c_{1}, c_{2}\right)$ is the angular kernel function. In the angle basis, $\hat{C}$ is diagonal in $l$ and $m$, a fact that follows from the rotational symmetry of $C\left(\mathbf{c}_{1}, \mathbf{c}_{2}\right)$ [16].

We may now express the linearized $\mathrm{U}-\mathrm{U}$ equation as an operator equation. Without any loss of generality, we can assume that the wave vector $\mathbf{k}=k \hat{e}_{z}$, where $\hat{e}_{z}$ is a unit vector along the $z$ direction. The U-U equation then takes the form

$$
-i \omega|\phi\rangle+i v_{T} k \hat{c}_{z}|\phi\rangle=-\gamma \hat{C}|\phi\rangle,
$$

where $\hat{c}_{z}$ is now an operator defined by $\hat{c}_{z}|\mathbf{c}\rangle=c_{z}|\mathbf{c}\rangle$. This form of U-U equation allows us to determine the microscopic frequencies as a perturbation expansion in powers of $k$.

\section{B. Spectrum of the collision operator}

The spectrum of the collision operator plays a fundamental role in both the derivation of the hydrodynamic equations and the calculation of transport coefficients. By inspection, we can see that the collision operator has five zero eigenvalues, which correspond to the five conserved quantities (particle number, momentum, and energy) in a two-body collision. The nonzero eigenvalues are all positive and, for hard sphere or contact interactions, approach a finite limiting value $\lambda_{M}=\frac{\sigma_{3}}{z}[12,16]$.

The representation of $\hat{C}$ in the angle basis shows that the eigenfunctions of the collision operator are states of definite $l$ and $m$. This allows us to use the indices $n, l, m$ to label the eigenfunctions $\left|\phi_{n, l, m}\right\rangle$ of $\hat{C}$ and write

$$
\left\langle c^{\prime}, l^{\prime}, m^{\prime} \mid \phi_{n, l, m}\right\rangle=\phi_{n, l}\left(c^{\prime}\right) \delta_{l, l^{\prime}} \delta_{m, m^{\prime}}
$$

where $\phi_{n, l}(c)$ is the radial part of the eigenfunction. If we define $\phi_{n, l, m}(c, \theta, \varphi)=\left\langle\mathbf{c} \mid \phi_{n, l, m}\right\rangle$, where $|\mathbf{c}\rangle=|c, \theta, \varphi\rangle$ in spherical coordinates, then the radial part of the eigenfunction is given by

$$
\phi_{n, l}(c)=c \sqrt{w(c)} \int d \Omega Y_{l}^{* m}(\theta, \varphi) \phi_{n, l, m}(c, \theta, \varphi) .
$$

For example, the particle number conservation eigenfunction is $\phi_{0,0,0}(\mathbf{c})=1$ and the radial part is $\phi_{0,0}(c)=c \sqrt{4 \pi w(c)}$. The radial part of the eigenfunctions satisfy the orthogonality condition

$$
\int_{0}^{\infty} d c \phi_{n^{\prime}, l}^{*}(c) \phi_{n, l}(c)=\delta_{n^{\prime}, n}
$$

\section{RELATION OF TRANSPORT COEFFICIENTS TO EIGENVALUES}

In order to relate the transport coefficients to the eigenvalues of the collision operator, we will equate frequencies obtained from the linearized hydrodynamic equations to frequencies derived directly from the U-U equation.

\section{A. Hydrodynamic frequencies}

A derivation of the linearized hydrodynamic equations and the hydrodynamic normal mode frequencies for a dilute fluid at temperatures above the critical temperature for BoseEinstein condensation can be found in Ref. [14]. For such a fluid there are five microscopically conserved quantities and therefore five hydrodynamic normal modes. The normal mode frequencies are as follows.

$$
\begin{aligned}
& \omega_{1}=\omega_{2}=-\frac{i k^{2} \eta}{m n_{0}}, \\
& \omega_{3}=-\frac{i k^{2} \kappa}{n_{0} c_{p}}, \\
& \omega_{4}=-k c_{s}-\frac{i k^{2}}{2 m n_{0}}\left[\zeta+\frac{4}{3} \eta+\frac{4 m \kappa \sigma_{2}}{15 k_{B} \sigma_{4}}\right], \\
& \omega_{5}=k c_{s}-\frac{i k^{2}}{2 m n_{0}}\left[\zeta+\frac{4}{3} \eta+\frac{4 m \kappa \sigma_{2}}{15 k_{B} \sigma_{4}}\right],
\end{aligned}
$$

where $\eta$ is the shear viscosity, $\kappa$ is the thermal conductivity, and $\zeta$ is the bulk viscosity. The speed of sound is given by

$$
c_{s}=\sqrt{\frac{5 k_{B} T \sigma_{4}}{3 m \sigma_{2}}},
$$

and the specific heats at constant pressure and constant density are given by

$$
c_{p}=\frac{5 k_{B} \sigma_{0} \sigma_{4}}{2 \sigma_{2}^{2}}\left(\frac{5 \sigma_{0} \sigma_{4}-3 \sigma_{2}^{2}}{2 \sigma_{0} \sigma_{2}}\right)
$$

and

$$
c_{n}=\frac{3 k_{B}}{2}\left(\frac{5 \sigma_{0} \sigma_{4}-3 \sigma_{2}^{2}}{2 \sigma_{0} \sigma_{2}}\right) \text {, }
$$

respectively.

\section{B. Microscopic frequencies}

To obtain microscopic expressions for the hydrodynamic frequencies, we apply standard Rayleigh-Schroedinger degenerate perturbation theory to the Eq. (18), using the wave vector $k$ as the small parameter and $v_{T} k \hat{c}_{z}$ as the perturbation. As a first step, we expand $\omega$ and $|\phi\rangle$ in powers of $k$ so that $\omega=\omega^{(0)}+k \omega^{(1)}+k^{2} \omega^{(2)}+\cdots$ and $|\phi\rangle=\left|\phi^{(0)}\right\rangle+k\left|\phi^{(1)}\right\rangle+$ $k^{2}\left|\phi^{(2)}\right\rangle+\cdots$ and we then substitute these equations into Eq. (18) and require that the coefficients of each power of $k$ vanish separately.

In the limit when $k=0$, Eq. (18) can be written

$$
-i \omega_{\beta}^{(0)}\left|\phi_{\beta}^{(0)}\right\rangle=-\gamma \hat{C}\left|\phi_{\beta}^{(0)}\right\rangle \text {. }
$$

From this it is clear that the unperturbed eigenvectors $\left|\phi_{\beta}^{(0)}\right\rangle$ are just the eigenvectors of the collision operator $\hat{C}$. Let us denote the eigenvalues of the collision operator as $\lambda_{\beta}$ so that

$$
\hat{C}\left|\phi_{\beta}^{(0)}\right\rangle=\lambda_{\beta}\left|\phi_{\beta}^{(0)}\right\rangle \text {. }
$$

We will denote the five degenerate "zero" eigenvalues of $\hat{C}$ by $\beta=1, \ldots, 5$. The remaining positive nondegenerate eigenvalues of $\hat{C}$ will be denoted by $\beta=6, \ldots, \infty$. The five 
eigenvectors of $\hat{C}$ with $\beta=1, \ldots, 5$ represent microscopically conserved quantities. When properly normalized [using Eq. (12)] they are given by

$$
\begin{aligned}
\left\langle\mathbf{c} \mid \phi_{1}^{(0)}\right\rangle & =1, \\
\left\langle\mathbf{c} \mid \phi_{2}^{(0)}\right\rangle & =\frac{2 \sigma_{0}}{\sqrt{3\left(5 \sigma_{4} \sigma_{0}-3 \sigma_{2}^{2}\right)}}\left(c^{2}-\frac{3 \sigma_{2}}{2 \sigma_{0}}\right), \\
\left\langle\mathbf{c} \mid \phi_{3}^{(0)}\right\rangle & =\sqrt{\frac{2 \sigma_{0}}{\sigma_{2}}} c_{x}, \\
\left\langle\mathbf{c} \mid \phi_{4}^{(0)}\right\rangle & =\sqrt{\frac{2 \sigma_{0}}{\sigma_{2}}} c_{y}, \\
\left\langle\mathbf{c} \mid \phi_{5}^{(0)}\right\rangle & =\sqrt{\frac{2 \sigma_{0}}{\sigma_{2}}} c_{z} .
\end{aligned}
$$

Since $\lambda_{\beta}=0(\beta=1, \ldots, 5)$, we must apply degenerate perturbation theory $[14,17]$ to determine the linear combinations of $\left|\phi_{\beta}^{(0)}\right\rangle(\beta=1, \ldots, 5)$ appropriate for a convergent perturbation expansion. They are

$$
\begin{aligned}
\left|\psi_{1}^{(0)}\right\rangle & =\left|\phi_{3}^{(0)}\right\rangle \\
\left|\psi_{2}^{(0)}\right\rangle & =\left|\phi_{4}^{(0)}\right\rangle \\
\left|\psi_{3}^{(0)}\right\rangle & =-\sqrt{1-\alpha^{2}}\left|\phi_{1}^{(0)}\right\rangle+\alpha\left|\phi_{2}^{(0)}\right\rangle \\
\left|\psi_{4}^{(0)}\right\rangle & =\frac{1}{\sqrt{2}}\left(-\alpha\left|\phi_{1}^{(0)}\right\rangle-\sqrt{1-\alpha^{2}}\left|\phi_{2}^{(0)}\right\rangle+\left|\phi_{5}^{(0)}\right\rangle\right) \\
\left|\psi_{5}^{(0)}\right\rangle & =\frac{1}{\sqrt{2}}\left(\alpha\left|\phi_{1}^{(0)}\right\rangle+\sqrt{1-\alpha^{2}}\left|\phi_{2}^{(0)}\right\rangle+\left|\phi_{5}^{(0)}\right\rangle\right)
\end{aligned}
$$

where $\alpha=\sqrt{3 \sigma_{2}^{2} /\left(5 \sigma_{0} \sigma_{4}\right)}$. For $\beta>6,\left|\psi_{\beta}^{(0)}\right\rangle=\left|\phi_{\beta}^{(0)}\right\rangle$.

The perturbation expansion for the eigenfrequencies of the linearized U-U equation can now be written

$$
\begin{aligned}
\omega_{\beta}= & -i \gamma \lambda_{\beta}+v_{T} k\left\langle\psi_{\beta}^{(0)}\left|\hat{c}_{z}\right| \psi_{\beta}^{(0)}\right\rangle \\
& -\frac{i v_{T}^{2} k^{2}}{\gamma} \sum_{\beta^{\prime}, \lambda_{\beta^{\prime}} \neq \lambda_{\beta}} \frac{\left\langle\psi_{\beta}^{(0)}\left|\hat{c}_{z}\right| \psi_{\beta^{\prime}}^{(0)}\right\rangle\left\langle\psi_{\beta^{\prime}}^{(0)}\left|\hat{c}_{z}\right| \psi_{\beta}^{(0)}\right\rangle}{\lambda_{\beta^{\prime}}-\lambda_{\beta}}+\cdots
\end{aligned}
$$

We will only need to consider the hydrodynamic modes $\beta=$ $1, \ldots, 5$. The restriction in Eq. (30) that $\lambda_{\beta^{\prime}} \neq \lambda_{\beta}$ indicates that $\beta^{\prime} \geqslant 6$. With these considerations, we may rewrite Eq. (30) as

$$
\begin{aligned}
\omega_{\beta}= & v_{T} k\left\langle\psi_{\beta}^{(0)}\left|\hat{c}_{z}\right| \psi_{\beta}^{(0)}\right\rangle \\
& -\frac{i v_{T}^{2} k^{2}}{\gamma} \sum_{\beta^{\prime}=6}^{\infty} \frac{\left\langle\psi_{\beta}^{(0)}\left|\hat{c}_{z}\right| \psi_{\beta^{\prime}}^{(0)}\right\rangle\left\langle\psi_{\beta^{\prime}}^{(0)}\left|\hat{c}_{z}\right| \psi_{\beta}^{(0)}\right\rangle}{\lambda_{\beta^{\prime}}}+\cdots
\end{aligned}
$$

Since the eigenvalues $\lambda_{\beta^{\prime}}$ are all positive, the term of order $k^{2}$ lies on the negative imaginary axis and the hydrodynamic modes to decay towards global equilibrium.

\section{First-order corrections}

The first-order corrections to the hydrodynamic frequencies are given by

$$
\omega_{\beta}^{(1)}=v_{T} k\left\langle\psi_{\beta}^{(0)}\left|\hat{c}_{z}\right| \psi_{\beta}^{(0)}\right\rangle .
$$

It is straightforward to compute these quantities in the angle basis and we find

$$
\omega_{1}^{(1)}=\omega_{2}^{(1)}=\omega_{3}^{(1)}=0 \quad \text { and } \quad-\omega_{4}^{(1)}=\omega_{5}^{(1)}=v_{T} k \sqrt{\frac{5 \sigma_{4}}{6 \sigma_{2}}} .
$$

The frequencies $\omega_{4}^{(1)}$ and $\omega_{5}^{(1)}$ correspond to sound waves traveling at the speed $c_{s}$, which matches the result (23) obtained from hydrodynamics.

\section{Second-order corrections}

The second-order corrections to the hydrodynamic frequencies $\omega_{\beta}(\beta=1, \ldots, 5)$ are given by

$$
\omega_{\beta}^{(2)}=-\frac{i v_{T}^{2} k^{2}}{\gamma} \sum_{\beta^{\prime}=6}^{\infty} \frac{\left\langle\psi_{\beta}^{(0)}\left|\hat{c}_{z}\right| \psi_{\beta^{\prime}}^{(0)}\right\rangle\left\langle\psi_{\beta^{\prime}}^{(0)}\left|\hat{c}_{z}\right| \psi_{\beta}^{(0)}\right\rangle}{\lambda_{\beta^{\prime}}} .
$$

This expression is only of practical value when a small number of terms are needed for the sum to converge. In the case of the classical Maxwell gas, this is guaranteed because only a limited number of inner products $\left\langle\psi_{\beta^{\prime}}^{(0)}\left|\hat{c}_{z}\right| \psi_{\beta}^{(0)}\right\rangle$ are nonzero. For other classical soft potentials, the convergence comes from the steadily increasing $\lambda_{\beta^{\prime}}$.

When the eigenvalue spectrum has a cutoff, as it does for hard spheres and contact interactions [16], there are an infinite number of terms with $\lambda_{\beta^{\prime}} \approx \lambda_{M}$, and when they are summed they give a finite contribution to the transport coefficient. We can rearrange the summation in Eq. (34) to simplify the computation of the large number of terms that must be included. We rewrite Eq. (34) in the form

$$
\begin{aligned}
\omega_{\beta}^{(2)}= & -\frac{i v_{T}^{2} k^{2}}{\gamma}\left[\frac{\left\langle\psi_{\beta}^{(0)}\left|\hat{c}_{z}^{2}\right| \psi_{\beta}^{(0)}\right\rangle-\left\langle\psi_{\beta}^{(0)}\left|\hat{c}_{z}\right| \psi_{\beta}^{(0)}\right\rangle^{2}}{\lambda_{M}}\right. \\
& \left.+\sum_{\beta^{\prime},=6}^{\beta_{\max }} \frac{\left\langle\psi_{\beta}^{(0)}\left|\hat{c}_{z}\right| \psi_{\beta^{\prime}}^{(0)}\right\rangle\left\langle\psi_{\beta^{\prime}}^{(0)}\left|\hat{c}_{z}\right| \psi_{\beta}^{(0)}\right\rangle}{\mu_{\beta^{\prime}}}\right],
\end{aligned}
$$

where $\mu_{\beta^{\prime}}=\left(\frac{1}{\lambda_{\beta^{\prime}}}-\frac{1}{\lambda_{M}}\right)^{-1}$ and $\beta_{\max }$ is chosen so that $\lambda_{\beta_{\max }} \approx \lambda_{M}$. In contrast to Eq. (34), only the first few terms contribute to the sum in Eq. (35), because $\mu_{\beta^{\prime}}$ rapidly becomes large. As we will show in Sec. V, Eq. (35) must be used to obtain correct results for hard spheres and contact interactions. In Appendix A, we show in more detail how Eq. (35) is obtained from Eq. (34).

\section{E. Microscopic expressions for the transport coefficients}

We now equate the microscopic frequencies to the hydrodynamics frequencies. As we show in Appendix B, the transport coefficients can be written as

$$
\frac{\eta}{\xi}=\frac{\sigma_{4}}{2 \sigma_{3}}+\frac{8 \pi \sigma_{0}}{15 z} \sum_{n=0}^{n_{\max }^{(2)}} \frac{\left|Q_{n}\right|^{2}}{\mu_{n, 2}}
$$




$$
\begin{gathered}
\frac{2 m \kappa}{5 k_{B} \xi}=\frac{\left(7 \sigma_{6} \sigma_{2}-5 \sigma_{4}^{2}\right)}{4 \sigma_{2} \sigma_{3}}+\frac{8 \pi \sigma_{0}}{15 z} \sum_{n=1}^{n_{\max }^{(1)}} \frac{\left|R_{n}\right|^{2}}{\mu_{n, 1}}, \\
\frac{\zeta}{\xi}=\frac{8 \pi \sigma_{0}}{9 z} \sum_{n=2}^{n_{\max }^{(0)}} \frac{\left|S_{n}\right|^{2}}{\mu_{n, 0}},
\end{gathered}
$$

where $\xi=\frac{1}{8 \pi a^{2}} \sqrt{\frac{\pi m k_{B} T}{2}}$ and

$$
\begin{gathered}
Q_{n}=\int_{0}^{\infty} d c c^{3} \sqrt{w(c)} \psi_{n, 2}^{(0)}(c), \\
R_{n}=\int_{0}^{\infty} d c c^{2} \sqrt{w(c)}\left(c^{2}-\frac{5 \sigma_{4}}{2 \sigma_{2}}\right) \psi_{n, 1}^{(0)}(c), \\
S_{n}=\int_{0}^{\infty} d c c^{3} \sqrt{w(c)} \psi_{n, 0}^{(0)}(c) .
\end{gathered}
$$

The lower limits of the $n$ summations come from the condition that $\lambda_{n, l}>0$. The upper limits of the $n$ summations, $n_{\max }^{(l)}$, are the analogy to $\beta_{\max }$ for a specific value of $l$.

The expression for the bulk viscosity $\zeta$ implies that it is zero, because $S_{n}$ is identically zero for $n \geqslant 2$. This is easily seen if we note that $c^{3} \sqrt{w(c)}$ is a linear combination of $\psi_{0,0}^{(0)}(c)$ and $\psi_{1,0}^{(0)}(c)$ [see Eq. (20)], and thus the function $c^{3} \sqrt{w(c)}$ is orthogonal to $\psi_{n, 0}^{(0)}(c)$ for $n \geqslant 2$. Only the two values $S_{0}$ and $S_{1}$ are nonzero, but these are not included in the sum because $\lambda_{0,0}$ and $\lambda_{1,0}$ are zero. The fact that a monatomic gas has zero bulk viscosity is well known $[18,19]$. Here we have shown that this result holds true in a quantum treatment of the noncondensed Bose gas.

\section{NUMERICAL COMPUTATION OF TRANSPORT COEFFICIENTS}

Numerical computation of transport coefficients requires a numerical computation of the eigenvalues and eigenvectors of the collision operator. We form a matrix representation of the collision operator and diagonalize it $[16,20]$. Our results are obtained by performing the calculation with several different matrix sizes, and extrapolating these results to an infinite matrix size when appropriate.

In Fig. 1 we show our numerical results for the transport coefficients of a degenerate noncondensed boson gas. The transport coefficients are plotted versus the reduced temperature $T / T_{C}$, where $T_{C}$ is the critical temperature for BoseEinstein condensation. Note that the transport coefficients have an overall dependence on $\sqrt{T}$, which is divided out in the dimensionless transport coefficients. We also plot the Eucken number, which is defined as $m \kappa /\left(\eta c_{n}\right)$, where $c_{n}$ is given in Eq. (25).

Filled circles (black) depict the "exact" U-U equation result of Eqs. (36) and (37) (with contact potential). The straight solid (blue) line depicts the U-U value as $T / T_{C} \rightarrow \infty$ (the Boltzmann limit). The straight dashed (red) line depicts the approximate classical result obtained by using only the lowest order sonine polynomial $[6,14]$. The open circles (gray) show the results obtained by using Eq. (34) instead of Eq. (35) and ignoring the cutoff behavior.

The transport coefficients for the degenerate boson gas are indistinguishable from the classical Boltzmann values
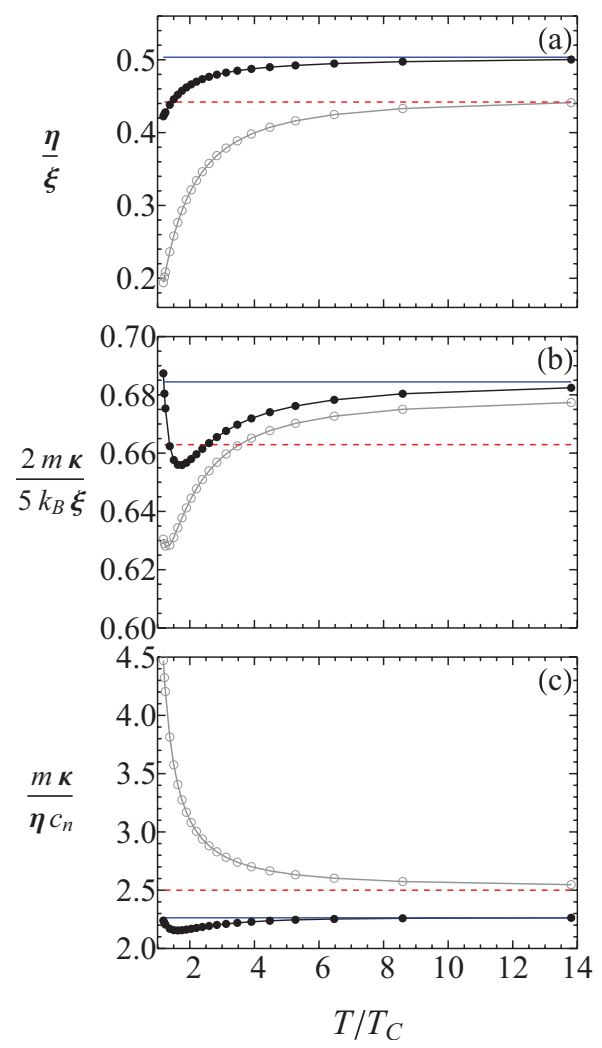

FIG. 1. (Color online) (a) Shear viscosity $\eta$, (b) thermal conductivity $\kappa$, and (c) the Eucken number versus temperature in dimensionless units. Solid circles (black) indicate the results of Eqs. (36) and (37), open circles (gray) show the results when Eq. (34) is used instead of Eq. (35). The straight dashed line (red) shows the classical lowest-order approximation using one Sonine polynomial and the straight solid line (blue) show the results of Eqs. (36) and (37) in the Boltzmann limit $T \rightarrow \infty$.

for temperatures above $20 T_{C}$, but become sensibly different for $T<10 T_{C}$. As one approaches $T_{C}$, the shear viscosity decreases to a value that is approximately $82 \%$ of its value at high temperature. The thermal conductivity shows a minimum around $T=1.5 T_{C}$ at approximately $95 \%$ of its high-temperature values and then increases as the temperature approaches $T_{C}$, perhaps anticipating the appearance of second sound [14,21] below $T_{C}$.

The Eucken number is useful for comparing our results to other systems and experiments, because it is independent of the overall interaction strength. To test the validity of our result, we compare Eucken numbers in the classical limit. We have calculated the Eucken number for soft potentials $\left(V(r) \sim r^{-n}\right)$, as a function of $n$, in the classical limit $\left(T / T_{C} \rightarrow \infty\right)$. This is accomplished by a using a generalization of the polynomial method for the Maxwell gas [20]. The results of this calculation are shown in Fig. 2. With a potential that goes as $r^{-4}$, the Eucken number is $5 / 2$. As the power of $1 / r$ is increased, the Eucken number slightly increases at first and then drops toward the hard sphere result (thick black line), which takes account of the cutoff. At very high powers of $1 / r$, increasingly large matrix sizes are needed to see convergence toward the hard 




FIG. 2. (Color online) Variation of the Eucken number with the power of $1 / r$ in the classical limit calculated with several matrix sizes. The thick solid line near the bottom shows the hard sphere result obtained by using Eq. (35). The thin solid line near the top shows the hard sphere result obtained when using Eq. (34). The dashed line (red) shows the classical lowest-order approximation using one Sonine polynomial and is exactly equal to $5 / 2$. The Eucken number for the Maxwell gas $\left(r^{-4}\right)$ is also exactly $5 / 2$.

sphere result. We find that the Eucken number of a true hard sphere gas is approximately 2.27 .

If Fig. 2, the thin line (gray) shows the hard sphere result when the cutoff behavior is ignored by directly using Eq. (34). The dashed line (red) shows the approximate hard sphere result of $5 / 2$ that is obtained by using only one Sonine polynomial. The Eucken number for soft potentials are close to both of these values, but as the potential becomes harder $(n \rightarrow \infty)$, it approaches the true hard sphere value.

It is interesting to note that experimentally observed Eucken numbers [22] for high-temperature dilute gases are much closer to $5 / 2$ than they are to the true hard sphere result. This should not be taken as validation of the "cutoff-ignoring" or "one Sonine" methods, because these systems in fact interact via a soft repulsive potential. One might consider using the observed Eucken number of the gas to estimate the power of $1 / r$ in the interaction potential.

These results are applicable to BECs because the lowenergy bosons interact via a contact potential [23], so the eigenvalues of the collision operator will have a cutoff, and one must be careful to include cutoff effects when computing transport properties.

\section{CONCLUSION}

We have derived an expression for the transport coefficients of a monatomic dilute Bose gas that obeys the UehlingUhlenbeck kinetic equation. Our expression relates the transport coefficients to a spectral decomposition of the linearized collision operator. This illuminates the relation between the collision operator, the microscopic hydrodynamic modes, and the transport coefficients.
We have calculated the transport coefficients of a Bose gas interacting with a contact potential as they vary with temperature. Overall, we find that the transport coefficients of the Bose gas decrease faster than a classical gas with decreasing temperature, owing to the increased scattering produced by the Bose enhancement factors in the collision integral. We also observe a sharp increase in the thermal conductivity as the temperature nears $T_{C}$, which may be a precursor of the onset of second sound in the condensate. Using the U-U equation instead of the Boltzmann equation does not change the fact the the bulk viscosity for a monatomic gas is zero.

Finally, we have shown that when an eigenvalue cutoff exists, as it does for contact interactions in the U-U collision operator, it is important to treat the cutoff correctly when calculating transport properties.

\section{ACKNOWLEDGMENTS}

The authors thank the Robert A. Welch Foundation (Grant No. F-1051) for support of this work.

\section{APPENDIX A: EXPRESSION FOR FREQUENCY CORRECTIONS IN THE PRESENCE OF AN EIGENVALUE CUTOFF}

In this appendix, we demonstrate how Eq. (35) is derived from Eq. (34). We begin with the crucial assumption that beyond a certain order $\left(\beta^{\prime}\right)$ the numerically calculated eigenvalues/eigenvectors become erroneous due to truncation errors in the matrix representation of the collision operator. The highest reliable eigenvalue/eigenvector pair will be labeled with $\beta_{\max }$. The rapid convergence of eigenvalues to their limiting value makes it acceptable to replace the remaining eigenvalues with the limiting value $\lambda_{M}$.

We seek a form of Eq. (34) that does not explicitly depend on the eigenvalues or eigenvectors with $\beta^{\prime}>\beta_{\max }$. To do this, we first split the sum in Eq. (34) into two parts,

$$
\begin{aligned}
\omega_{\beta}^{(2)}= & -\frac{i v_{T}^{2} k^{2}}{\gamma}\left[\sum_{\beta^{\prime}=6}^{\beta_{\max }} \frac{\left\langle\psi_{\beta}^{(0)}\left|\hat{c}_{z}\right| \psi_{\beta^{\prime}}^{(0)}\right\rangle\left\langle\psi_{\beta^{\prime}}^{(0)}\left|\hat{c}_{z}\right| \psi_{\beta}^{(0)}\right\rangle}{\lambda_{\beta^{\prime}}}\right. \\
& \left.+\frac{1}{\lambda_{M}} \sum_{\beta^{\prime}>\beta_{\max }}^{\infty}\left\langle\psi_{\beta}^{(0)}\left|\hat{c}_{z}\right| \psi_{\beta^{\prime}}^{(0)}\right\rangle\left\langle\psi_{\beta^{\prime}}^{(0)}\left|\hat{c}_{z}\right| \psi_{\beta}^{(0)}\right\rangle\right],
\end{aligned}
$$

where the first term accounts for the eigenmodes which are given accurately from the numerical calculation, and the second term contains all higher-order eigenmodes, which are not accurately determined by the numerical calculation.

To simplify the second term on the right-hand side of Eq. (A1), we rewrite it as

$$
\begin{aligned}
& \sum_{\beta^{\prime}>\beta_{\max }}^{\infty}\left\langle\psi_{\beta}^{(0)}\left|\hat{c}_{z}\right| \psi_{\beta^{\prime}}^{(0)}\right\rangle\left\langle\psi_{\beta^{\prime}}^{(0)}\left|\hat{c}_{z}\right| \psi_{\beta}^{(0)}\right\rangle \\
& =\sum_{\beta^{\prime \prime}=1}^{\infty}\left\langle\psi_{\beta}^{(0)}\left|\hat{c}_{z}\right| \psi_{\beta^{\prime \prime}}^{(0)}\right\rangle\left\langle\psi_{\beta^{\prime \prime}}^{(0)}\left|\hat{c}_{z}\right| \psi_{\beta}^{(0)}\right\rangle
\end{aligned}
$$




$$
\begin{aligned}
& -\sum_{\beta^{\prime \prime}=1}^{5}\left\langle\psi_{\beta}^{(0)}\left|\hat{c}_{z}\right| \psi_{\beta^{\prime \prime}}^{(0)}\right\rangle\left\langle\psi_{\beta^{\prime \prime}}^{(0)}\left|\hat{c}_{z}\right| \psi_{\beta}^{(0)}\right\rangle \\
& -\sum_{\beta^{\prime \prime}=6}^{\beta_{\max }}\left\langle\psi_{\beta}^{(0)}\left|\hat{c}_{z}\right| \psi_{\beta^{\prime \prime}}^{(0)}\right\rangle\left\langle\psi_{\beta^{\prime \prime}}^{(0)}\left|\hat{c}_{z}\right| \psi_{\beta}^{(0)}\right\rangle .
\end{aligned}
$$

The first term is identified as $\left\langle\psi_{\beta}^{(0)}\left|\hat{c}_{z}^{2}\right| \psi_{\beta}^{(0)}\right\rangle$, due to the fact that the eigenvectors $\left|\psi_{\beta^{\prime \prime}}^{(0)}\right\rangle$ with $\beta^{\prime \prime}=1, \ldots \infty$ form a complete set. The second term is identified as $\left\langle\psi_{\beta}^{(0)}\left|\hat{c}_{z}\right| \psi_{\beta}^{(0)}\right\rangle^{2}$, due to the fact that the eigenvectors $\left|\psi_{\beta}^{(0)}\right\rangle$ satisfy $\left\langle\psi_{\beta}^{(0)}\left|\hat{c}_{z}\right| \psi_{\beta^{\prime \prime}}^{(0)}\right\rangle=$ $\delta_{\beta, \beta^{\prime \prime}}\left\langle\psi_{\beta}^{(0)}\left|\hat{c}_{z}\right| \psi_{\beta}^{(0)}\right\rangle$ for $\beta^{\prime \prime}=1, \ldots 5$. The third term can be combined with the first term of Eq. (A1).

Using these simplifications, we obtain

$$
\begin{aligned}
\omega_{\beta}^{(2)}= & -\frac{i v_{T}^{2} k^{2}}{\gamma}\left[\frac{\left\langle\psi_{\beta}^{(0)}\left|\hat{c}_{z}^{2}\right| \psi_{\beta}^{(0)}\right\rangle-\left\langle\psi_{\beta}^{(0)}\left|\hat{c}_{z}\right| \psi_{\beta}^{(0)}\right\rangle^{2}}{\lambda_{M}}\right. \\
& \left.+\sum_{\beta^{\prime}=6}^{\beta_{\max }} \frac{\left\langle\psi_{\beta}^{(0)}\left|\hat{c}_{z}\right| \psi_{\beta^{\prime}}^{(0)}\right\rangle\left\langle\psi_{\beta^{\prime}}^{(0)}\left|\hat{c}_{z}\right| \psi_{\beta}^{(0)}\right\rangle}{\mu_{\beta^{\prime}}}\right]
\end{aligned}
$$

where $\mu_{\beta^{\prime}}=\left(\frac{1}{\lambda_{\beta^{\prime}}}-\frac{1}{\lambda_{M}}\right)^{-1}$.

This approximate expression for $\omega_{\beta}^{(2)}$ does not depend on any of the unreliable eigenmodes or eigenvalues. It is approximate because in deriving it, we have replaced a portion of the eigenvalues with $\lambda_{M}$. The appropriateness of this replacement may be judged by examining the values $\mu_{\beta^{\prime}}$, which quickly become large as the eigenvalues approach $\lambda_{M}$. A negative value of $\mu_{\beta^{\prime}}$ indicates that $\lambda_{\beta^{\prime}}$ is erroneous and we use this as the condition for determining $\beta_{\max }$.

\section{APPENDIX B: CALCULATION OF FREQUENCY CORRECTIONS}

In this appendix, we give some details of the calculations needed to obtain Eqs. (36), (37) and (38) from the general expression Eq. (35).

The second-order corrections to the hydrodynamic frequencies can be written as

$$
\omega_{\beta}^{(2)}=-\frac{i v_{T}^{2} k^{2}}{\gamma}\left[\frac{\Delta_{\beta}}{\lambda_{M}}+\Omega_{\beta}\right]
$$

where we have defined

$$
\Delta_{\beta}=\left\langle\psi_{\beta}^{(0)}\left|\hat{c}_{z}^{2}\right| \psi_{\beta}^{(0)}\right\rangle-\left\langle\psi_{\beta}^{(0)}\left|\hat{c}_{z}\right| \psi_{\beta}^{(0)}\right\rangle^{2},
$$

and

$$
\Omega_{\beta}=\sum_{\beta^{\prime}=6}^{\beta_{\max }} \frac{\left\langle\psi_{\beta}^{(0)}\left|\hat{c}_{z}\right| \psi_{\beta^{\prime}}^{(0)}\right\rangle\left\langle\psi_{\beta^{\prime}}^{(0)}\left|\hat{c}_{z}\right| \psi_{\beta}^{(0)}\right\rangle}{\mu_{\beta^{\prime}}} .
$$

Or, more explicitly,

$$
\Omega_{\beta}=\sum_{l, m} \sum_{n, \lambda_{n, l}>0}^{\lambda_{n, l}<\lambda_{M}} \frac{\left\langle\psi_{\beta}^{(0)}\left|\hat{c}_{z}\right| \psi_{n, l, m}^{(0)}\right\rangle\left\langle\psi_{n, l, m}^{(0)}\left|\hat{c}_{z}\right| \psi_{\beta}^{(0)}\right\rangle}{\mu_{n, l}} .
$$

Simplification of Eqs. (B2) and (B4) can be done relatively quickly by using the angle basis defined in Sec. III A. First we must express the zeroth-order eigenstates in terms of the $|c, l, m\rangle$ basis. They are

$$
\begin{aligned}
& \left\langle c, l, m \mid \psi_{1}^{0}\right\rangle=\sqrt{\frac{4 \pi \sigma_{0} w(c)}{3 \sigma_{2}}} c^{2} \delta_{l, 1}\left(\delta_{m, 1}-\delta_{m,-1}\right), \\
& \left\langle c, l, m \mid \psi_{2}^{0}\right\rangle=i \sqrt{\frac{4 \pi \sigma_{0} w(c)}{3 \sigma_{2}}} c^{2} \delta_{l, 1}\left(\delta_{m, 1}+\delta_{m,-1}\right), \\
& \left\langle c, l, m \mid \psi_{3}^{0}\right\rangle=\frac{2 \sigma_{2}}{5 \sigma_{4} s_{2}} c \sqrt{4 \pi w(c)}\left(c^{2}-\frac{5 \sigma_{4}}{2 \sigma_{2}}\right) \delta_{l, 0} \delta_{m, 0}, \\
& \left\langle c, l, m \mid \psi_{4}^{0}\right\rangle=c \sqrt{\frac{4 \pi \sigma_{0} w(c)}{3 \sigma_{2}}}\left(-\sqrt{\frac{2 \sigma_{2}}{5 \sigma_{4}}} c^{2} \delta_{l, 0}+c \delta_{l, 1}\right) \delta_{m, 0}, \\
& \left\langle c, l, m \mid \psi_{5}^{0}\right\rangle=c \sqrt{\frac{4 \pi \sigma_{0} w(c)}{3 \sigma_{2}}}\left(\sqrt{\frac{2 \sigma_{2}}{5 \sigma_{4}}} c^{2} \delta_{l, 0}+c \delta_{l, 1}\right) \delta_{m, 0} .
\end{aligned}
$$

Expressing the operator $\hat{c}_{z}$ in the $|c, l, m\rangle$ basis provides and elegant an economical way to evaluate $\Delta_{\beta}$ and $\Omega_{\beta}$. We can do this by inserting identity operators to obtain

$$
\begin{aligned}
\hat{c}_{z}= & \int_{0}^{\infty} d c \sum_{l, m} \int_{0}^{\infty} d c^{\prime} \sum_{l^{\prime}, m^{\prime}} \int d \mathbf{c}_{1}|c, l, m\rangle \\
& \times\left\langle c, l, m\left|\hat{c}_{z}\right| \mathbf{c}_{1}\right\rangle\left\langle\mathbf{c}_{1} \mid c^{\prime}, l^{\prime}, m^{\prime}\right\rangle\left\langle c^{\prime}, l^{\prime}, m^{\prime}\right| .
\end{aligned}
$$

We then use the definitions (15) to get

$$
\begin{aligned}
\hat{c}_{z}= & \int_{0}^{\infty} d c \sum_{l, m} \sum_{l^{\prime}, m^{\prime}}|c, l, m\rangle \\
& \times\left[\int d \Omega c_{z} Y_{l}^{* m}(\theta, \phi) Y_{l^{\prime}}^{m^{\prime}}(\theta, \phi)\right]\left\langle c, l^{\prime}, m^{\prime}\right|
\end{aligned}
$$

Performing the angular integration in the bracketed term involves Wigner 3-j symbols, but can be simplified to

$$
\begin{aligned}
\hat{c}_{z}= & \int_{0}^{\infty} d c c \sum_{l=0}^{\infty} \sum_{m=-l}^{l}|c, l, m\rangle \\
& \times\left(J_{l+1, m}\langle c, l+1,-m|+J_{l, m}\langle c, l-1,-m|\right),
\end{aligned}
$$

where $J_{l, m}=\sqrt{\frac{(l-m)(l+m)}{(2 l-1)(2 l+1)}}$. We can also obtain a compact expression for $\hat{c}_{z}^{2}$ in the angle basis.

Calculating $\Delta_{\beta}$ using the expressions (B5) and (B8), one obtains

$$
\begin{gathered}
\Delta_{1}=\Delta_{2}=\frac{\sigma_{4}}{2 \sigma_{2}}, \\
\Delta_{3}=\frac{\sigma_{2}}{2 \sigma_{4}} \frac{7 \sigma_{6} \sigma_{2}-5 \sigma_{4}^{2}}{5 \sigma_{0} \sigma_{4}-3 \sigma_{2}^{2}}, \\
\Delta_{4}=\Delta_{5}=\frac{7 \sigma_{6} \sigma_{2}-\sigma_{4}^{2}}{12 \sigma_{2} \sigma_{4}} .
\end{gathered}
$$

To demonstrate the method of calculation for $\Omega_{\beta}$, we outline the calculation of $\Omega_{1}$ below. Starting with Eq. (B4) with $\beta=1$, 
we begin by inserting the expression (B8) for both occurrences of $\hat{c}_{z}$ and using the relations in Eqs. (B5) and (19) to obtain

$$
\begin{aligned}
\Omega_{1}= & \frac{4 \pi \sigma_{0}}{3 \sigma_{2}} \sum_{n, \lambda_{n, l}>0}^{n_{\max }^{(l)}} \sum_{l, m} \frac{1}{\mu_{n, l}} \sum_{l_{1}, m_{1}} \sum_{l_{2}, m_{2}} \\
& \times \int_{0}^{\infty} d c_{1} c_{1}^{3} \sqrt{w\left(c_{1}\right)} \psi_{n, l}\left(c_{1}\right) \int_{0}^{\infty} d c_{2} c_{2}^{3} \sqrt{w\left(c_{2}\right)} \psi_{n, l}^{*}\left(c_{2}\right) \\
& \times \delta_{l_{1}, 1}\left(\delta_{m_{1}, 1}-\delta_{m_{1},-1}\right) \delta_{m,-m_{1}} \delta_{l, l_{2}}\left(\delta_{m_{2},-1}-\delta_{m_{2}, 1}\right) \delta_{m, m_{2}} \\
& \times\left(J_{l_{1}+1, m_{1}} \delta_{l, l_{1}+1}+J_{l_{1}, m_{1}} \delta_{l, l_{1}-1}\right)\left(J_{l_{2}+1, m_{2}} \delta_{l_{2}+1,1}\right. \\
& \left.+J_{l_{2}, m_{2}} \delta_{l_{2}-1,1}\right) .
\end{aligned}
$$

Performing the summations and some minor simplifications, we get

$$
\Omega_{1}=\frac{8 \pi \sigma_{0}}{15 \sigma_{2}} \sum_{n=0}^{n_{\max }^{(2)}} \frac{\left|Q_{n}\right|^{2}}{\mu_{n, 2}}
$$

where $Q_{n}$ is given in Eq. (39). In a similar calculation, we find that $\Omega_{2}=\Omega_{1}$ and that

$$
\begin{gathered}
\Omega_{3}=\frac{16 \pi \sigma_{2}^{2} \sigma_{0}}{15 \sigma_{4}\left(5 \sigma_{4} \sigma_{0}-3 \sigma_{2}^{2}\right)} \sum_{n=1}^{n_{\max }^{(1)}} \frac{\left|R_{n}\right|^{2}}{\mu_{n, 1}}, \\
\Omega_{4}=\Omega_{5}=\frac{8 \pi \sigma_{0}}{45 \sigma_{4}} \sum_{n=1}^{n_{\max }^{(1)}} \frac{\left|R_{n}\right|^{2}}{\mu_{n, 1}} \\
+\frac{16 \pi \sigma_{0}}{45 \sigma_{2}} \sum_{n=0}^{n_{\max }^{(2)}} \frac{\left|Q_{n}\right|^{2}}{\mu_{n, 2}}+\frac{4 \pi \sigma_{0}}{9 \sigma_{2}} \sum_{n=2}^{n_{\max }^{(0)}} \frac{\left|S_{n}\right|^{2}}{\mu_{n, 0}},
\end{gathered}
$$

where $R_{n}$ and $S_{n}$ are those given in Eqs. (40) and (41).

In carrying out the calculation of $\Omega_{\beta}$ for this five cases $(\beta=$ $1, \ldots, 5)$, we find that there is only one remaining summation. The range of this summation must be restricted so that $0<$ $\lambda_{n, l}<\lambda_{M}$. We define $n_{\max }^{(l)}$ in analogy with $\beta_{\max }$ as the largest value of $n$ for which the numerically computed $\lambda_{n, l}$ is less than $\lambda_{M}$ for a given value of $l$.
[1] S. Kikuchi and L. Nordheim, Zeits. f. Physik 60, 652 (1930).

[2] E. A. Uehling and G. E. Uhlenbeck, Phys. Rev. 43, 552 (1933).

[3] Sangyong Jeon, Phys. Rev. D 52, 3591 (1995).

[4] D. Davesne, Phys. Rev. C 53, 3069 (1996).

[5] T. Nikuni and A. Griffin, Phys. Rev. A 63, 033608 (2001).

[6] S. Chapman and T. G. Cowling, Mathematical Theory of NonUniform Gases (Cambridge University Press, Cambridge, 1970).

[7] P. Résibois, J. Stat. Phys. 2, 21 (1970).

[8] Antonio Dobado and Felipe J. Llanes-Estrada, Phys. Rev. D 69, 116004 (2004).

[9] P. K. Kovtun, D. T. Son, and A. O. Starinets, Phys. Rev. Lett. 94, 111601 (2005).

[10] K. Dusling and D. Teaney, Phys. Rev. C 77, 034905 (2008).

[11] T. Enss, C. Küppersbusch, and L. Fritz, Phys. Rev. A 86, 013617 (2012).

[12] I. Kuščer and M. M. R. Williams, Phys. Fluids 10, 1922 (1967).

[13] Byung Chan Eu, Transport Coefficients of Fluids (SpringerVerlag, Berlin, 2006).
[14] L. E. Reichl, A Modern Course in Statistical Physics, 3rd ed. (Wiley-VCH, Weinheim, 2009).

[15] E. D. Gust and L. E. Reichl, arXiv:1202.3418 (2012).

[16] E. D. Gust and L. E. Reichl, Phys. Rev. E 81, 061202 (2010).

[17] Eugene Merzbacher, Quantum Mechanics (Wiley, New York, 1961).

[18] Walter G. Vincenti and Charles H. Kruger, Jr., Introduction to Physical Gas Dynamics (Robert E. Krieger, Huntington, New York, 1967).

[19] Rick E. Graves and Brian M. Argrow, J. Thermophys. Heat Transfer 13, 337 (1999).

[20] E. D. Gust and L. E. Reichl, Phys. Rev. E 79, 031202 (2009).

[21] S. J. Putterman, Superfluid Hydrodynamics (North-Holland, Amsterdam, 1974).

[22] CRC Handbook of Chemistry and Physics, 93rd ed. (CRC Press, Boca Raton, 2012), pp. 6-21, 6-229, 6-240.

[23] C. J. Pethick and H. Smith, Bose-Einstein Condensation in Dilute Gases (Cambridge University Press, Cambridge, 2002). 